ELSEVIER

\title{
A Comparative, Randomized Trial of Concentration-Controlled Sirolimus Combined With Reduced-Dose Tacrolimus or Standard-Dose Tacrolimus in Renal Allograft Recipients
}

\author{
W.O. Bechstein, L. Paczek, L. Wramner, J.-P. Squifflet, and A.J. Zygmunt, for the European Rapamune \\ Tacrolimus Study Group
}

\begin{abstract}
Background. The clinical safety and efficacy of sirolimus plus reduced-dose tacrolimus was evaluated in de novo renal allograft recipients enrolled in a comparative, open-label study.

Methods. One hundred twenty-eight renal allograft recipients were randomly assigned (1:1) to receive reduced-dose tacrolimus plus sirolimus (rTAC) or standard-dose tacrolimus and sirolimus (sTAC) for 6 months. The primary efficacy endpoint was calculated creatinine clearance values at 6 months.

Results. Demographic variables were similar between groups. At 6 months, mean ( \pm standard deviation) calculated creatinine clearance was significantly improved in the rTAC group (63.8 vs $52.7 \mathrm{~mL} / \mathrm{min}, P=.005)$, although mean serum creatinine values were not significantly different. Patient survival $(95.2 \%$ and $96.9 \%)$ and graft survival $(93.7 \%$ and $98.5 \%$ ) were similar between the rTAC and sTAC groups, respectively. Acute rejection rates were $17.5 \%$ with rTAC and $7.7 \%$ with sTAC $(P=.095)$.

Conclusions. The rTAC regimen provided effective immunosuppression and was associated with improved creatinine clearance. Adequate immunosuppressant exposure must be achieved in the early postoperative period to minimize the risk of acute rejection.
\end{abstract}

$\mathbf{S}^{\mathrm{n}}$ NCE THEIR INTRODUCTION, the calcineurin inhibitors (CNIs) cyclosporine and tacrolimus have generally remained the cornerstones of immunosuppressive protocols. The use of CNIs, however, may be complicated by toxicities, including hypertension, post-transplant diabetes, hyperlipidemia, and nephrotoxicity. Toxicity with CNIs is known to contribute to a long-term decline in renal function and to the development of chronic allograft nephropathy. ${ }^{1-5}$ Because of these limitations, significant research efforts are directed toward developing immunosuppressive strategies that minimize or eliminate the use of CNIs. Immunosuppressive protocols using agents such as sirolimus (rapamycin; Rapamune) may reduce or eliminate chronic exposure to CNIs, with the potential benefit of improving renal allograft function and outcomes.

Sirolimus, a macrocyclic lactone isolated from Streptomyces hygroscopicus, is a potent immunosuppressive agent with a multifaceted mechanism of action distinct from that of CNIs. ${ }^{6}$ Sirolimus does not inhibit the activity of calcineurin phosphatase. It forms a complex with FKBP-12 that binds to the mammalian target of rapamycin, a specific cellcycle regulatory protein, inhibiting cytokine-induced signal transduction pathways and arresting the cell cycle in the G1-S phase. ${ }^{7}$ Sirolimus also inhibits various growth factors that are critical in regulating and inhibiting the proliferation and migration of vascular smooth muscle cells and has been

From the Ruhr-Universitat (W.O.B.), Bochum, Germany; Transplantation Institute (L.P.), Medical University of Warsaw, Poland; Transplantation Center (L.W.), Sahlgrenska University Hospital, Göteborg, Sweden; Centre Hospitalier Universitaire du Sart-Tilman (J.-P.S.), Liège, Belgium; Wyeth Research (A.J.Z.), Collegeville, Pennsylvania, USA.

At the time of this study, Anthony J. Zygmunt was an employee of Wyeth Research.

Address reprint requests to Wolf Otto Bechstein, MD, PhD, Department of General and Visceral Surgery, Goethe University Hospital, Senckenberganlage 31, 60325 Frankfurt am Main, Germany. E-mail: Wolf.Bechstein@kgu.de

$0041-1345 / 13 / \$$-see front matter http://dx.doi.org/10.1016/j.transproceed.2013.03.025 
shown to inhibit arterial intimal thickening in animal models and humans. ${ }^{8-12}$

Because sirolimus and tacrolimus share a common immunophilin (FKBP-12), the combination of sirolimus and tacrolimus was initially believed to be antagonistic. ${ }^{13,14}$ Early preclinical experience, however, indicated that the sirolimus-tacrolimus combination exhibited immunosuppressive synergy. ${ }^{15}$ Only a small fraction of the abundant FKBP-12 immunophilin needs to be occupied by these agents to achieve maximal immunosuppression. ${ }^{16}$ There is an expanding body of literature on the successful clinical application of the combination of sirolimus- and tacrolimusbased immunosuppression in renal and non-renal allograft recipients. $^{17-25}$

In an attempt to minimize the toxicity of tacrolimus, this study was designed to investigate the clinical safety and efficacy of a regimen of sirolimus plus reduced-dose tacrolimus (rTAC) in renal allograft recipients.

\section{METHODS}

This 6-month, randomized, open-label trial enrolled 128 de novo renal allograft recipients at 13 European sites. The study was conducted in accordance with guidelines established by the Declaration of Helsinki and was completed in June 2002. Approval was granted by the institutional review board or human ethics committee of each study center. Each enrolled patient provided written informed consent.

\section{Patient Population}

All patients $(n=128)$ were aged 18 years or older and received either a primary or secondary renal allograft from a deceased donor. Patients with secondary transplants must have maintained their primary graft for a minimum of 6 months to be eligible (with the exception of patients who had lost their primary graft within 6 months secondary to a technical complication). Women of childbearing potential were required to have a negative pregnancy test result before sirolimus administration and to use a medically acceptable method of contraception. Patients were excluded if they had a systemic infection, human immunodeficiency virus, active hepatitis $\mathrm{B}$ or $\mathrm{C}$, history of malignancy within the previous 5 years (except for adequately treated basal cell or squamous cell carcinoma of the skin), known hypersensitivity to sirolimus or tacrolimus or their derivatives, and a screening or baseline white blood cell count $\leq 3000 / \mathrm{mm}^{3}$ or platelet count $\leq 100,000 / \mathrm{mm}^{3}$. Use of an investigational drug or treatment within 4 weeks before enrollment or during the 6-month treatment phase was prohibited. Patients planning to use medications known to interact with sirolimus were excluded. Use of terfenadine, cisapride, astemizole, pimozide, or ketoconazole must have been discontinued before receiving sirolimus. Patients receiving multiple organ transplants, allografts with cold ischemia times longer than 36 hours, allografts obtained from donors after cardiac death, or allografts from donors older than 65 years were excluded. Patients at high risk for acute rejection (AR) were excluded, including those with recent panel-reactive antibodies $>50 \%$.

\section{Immunosuppressive Therapy}

Patients were randomly assigned 1:1 before transplantation to receive corticosteroids and sirolimus oral solution in combination with either rTAC or standard-dose tacrolimus (sTAC). All patients received corticosteroids according to a standardized taper regimen: post-transplant day (PTD) 0, $500 \mathrm{mg}$ methylprednisolone intravenously (IV); PTD 1, $125 \mathrm{mg}$ methylprednisolone IV; PTD 2 to week 2 , prednisone $20 \mathrm{mg}$ orally $(\mathrm{PO})$ daily; weeks 2 to 4 , prednisone $15 \mathrm{mg}$ PO daily; months 1 to 2, prednisone $10 \mathrm{mg}$ PO daily; months 3 to 6 prednisone $5 \mathrm{mg}$ PO daily. Planned antibody induction within 1 week before or at the time of transplantation was not permitted; however, the use of antibody therapy was allowed to manage suspected AR, steroid-resistant rejection, or delayed graft function. Concurrent use of other immunosuppressive therapies, including Neoral (cyclosporine), CellCept (mycophenolate mofetil), or azathioprine, was not allowed.

Administration of sirolimus oral solution $(1 \mathrm{mg} / \mathrm{mL})$ was initiated within 48 hours after transplantation. In the rTAC group, patients received an initial sirolimus loading dose of $15 \mathrm{mg}$ on day 1 , then $5 \mathrm{mg}$ daily adjusted to maintain 24-hour whole blood trough levels, assessed via high-performance liquid chromatography-mass spectometry (Table 1). Tacrolimus was initiated within 7 days after transplantation at a dose of $0.03 \mathrm{mg} / \mathrm{kg}$ twice daily, adjusted to maintain trough levels of 3 to $7 \mathrm{ng} / \mathrm{mL}$ throughout the study period.

In the sTAC group, patients received an initial sirolimus loading dose of $6 \mathrm{mg}$ on day 1 , then $2 \mathrm{mg}$ daily adjusted to maintain 24-hour whole blood trough levels of 5 to $10 \mathrm{ng} / \mathrm{mL}$ throughout the study. Tacrolimus was initiated within 7 days after transplantation at a dose of $0.05 \mathrm{mg} / \mathrm{kg}$ twice daily, adjusted to maintain target trough levels (Table 1).

Soon after study enrollment began, an increased incidence of AR was identified in the rTAC group. Analysis of this patient group revealed that target sirolimus and tacrolimus levels were not being achieved by day 7 . The protocol was therefore amended to mandate an increase in the sirolimus loading dose to $15 \mathrm{mg}$ for 3 days, and the initial sirolimus daily dose was increased to $5 \mathrm{mg}$ in both groups (Table 1). In the rTAC group, the initial tacrolimus dose was

Table 1. Dosing and Target Trough Concentrations of Sirolimus and Tacrolimus

\begin{tabular}{|c|c|c|c|c|}
\hline \multirow[b]{2}{*}{ Time point } & \multicolumn{2}{|l|}{ rTAC group } & \multicolumn{2}{|l|}{ sTAC group } \\
\hline & Sirolimus & Tacrolimus & Sirolimus & Tacrolimus \\
\hline Preamendment & $15 \mathrm{mg}$ (day 1), then $5 \mathrm{mg} \mathrm{QD}{ }^{\mathrm{a}}$ & $0.03 \mathrm{mg} / \mathrm{kg} \mathrm{BID}{ }^{\mathrm{a}}$ & $6 \mathrm{mg}$ (day 1 ), then $2 \mathrm{mg} \mathrm{QD}$ & $0.05 \mathrm{mg} / \mathrm{kg} \mathrm{BID}^{\mathrm{a}}$ \\
\hline Postamendment & $15 \mathrm{mg}$ for $3 \mathrm{~d}$, then $5 \mathrm{mg} \mathrm{QD}{ }^{\mathrm{a}}$ & $0.05 \mathrm{mg} / \mathrm{kg} \mathrm{BID}^{\mathrm{a}}$ & $15 \mathrm{mg}$ for 3 day, then $5 \mathrm{mg} \mathrm{QD}$ & $0.05 \mathrm{mg} / \mathrm{kg} \mathrm{BID}^{\mathrm{a}}$ \\
\hline \multicolumn{5}{|c|}{$\begin{array}{l}\text { Target trough concentrations } \\
\quad(\mathrm{ng} / \mathrm{mL})\end{array}$} \\
\hline Month 1 & $10-20$ & $3-7$ & $5-10$ & $10-15$ \\
\hline Months 2-3 & $10-15$ & $3-7$ & $5-10$ & $8-12$ \\
\hline$>$ Month 3 & $8-15$ & $3-7$ & $5-10$ & $8-12$ \\
\hline
\end{tabular}

BID, twice daily; rTAC, reduced-dose tacrolimus; sTAC, standard-dose tacrolimus; QD, once daily.

${ }^{\mathrm{a} D o s i n g}$ adjusted to maintain 24 -h whole blood trough levels. 
increased to $0.05 \mathrm{mg} / \mathrm{kg}$. Target sirolimus and tacrolimus concentrations were not changed.

\section{Required Concomitant Treatment}

Prophylaxis for Pneumocystis carinii pneumonia was required for all patients throughout the treatment period. Trimethoprimsulfamethoxazole was the preferred therapy, although alternative agents were permitted in accordance with local standards of care. Cytomegalovirus-negative recipients of an allograft from a cytomegalovirus-positive donor received cytomegalovirus prophylaxis for 3 months after transplantation, according to local practice.

\section{Acute Rejection}

Patients with clinically suspected AR underwent a biopsy to confirm the diagnosis, unless contraindicated. The histologic diagnosis and severity grade of AR was scored according to the 1997 Banff classification ${ }^{26}$ by a local pathologist who was blinded to treatment. Biopsy-confirmed AR (BCAR) included only those episodes in which the Banff AR grade was at least IA. Clinical AR was defined as all first BCARs plus those treated episodes in which a biopsy was not performed or was graded as borderline. Initial therapy for AR consisted of pulse corticosteroids, according to local standards of care. Patients who did not respond to corticosteroids could receive antibody therapy and were eligible to continue in the study at the discretion of the investigator. Patients who required other maintenance immunosuppressive agents were withdrawn from the study.

\section{Graft Loss}

Graft loss was defined as (1) a deterioration of renal function sufficient to require a transplant nephrectomy; (2) reinitiation of dialysis for more than 8 weeks; (3) retransplantation; or (4) death with a functioning graft.

\section{Endpoints}

The primary endpoint was graft function as assessed by calculated creatinine clearance using the method of Nankivell ${ }^{27}$ at 6 months posttransplant. Secondary efficacy endpoints at 6 months included graft function as assessed by serum creatinine, the incidence of BCARs and presumptive ARs, the time to first BCAR, the severity of AR (including histologic grade), patient and graft survival, the incidence of infection (confirmed, presumptive, and opportunistic), histologically confirmed lymphoproliferative disease or malignancy, new-onset diabetes mellitus, and premature withdrawal from study medication.

\section{Safety Assessment}

Safety was assessed via routine physical examinations, which included measurement of weight and vital signs, electrocardiograms, complete blood chemistries, blood counts, serum creatinine levels, calculated creatinine clearance, and fasting lipid profiles. Chest radiographs were performed before enrollment and as clinically indicated. All adverse events were recorded, and study participants were monitored for infections, malignancies, and lymphoproliferative disease.

\section{Statistical Analysis}

It was estimated that a sample size of 70 patients per group would have $90 \%$ power to detect a difference in mean serum creatinine as small as $27.5 \mu \mathrm{mol} / \mathrm{L}$, assuming a standard deviation (SD) of 50 , with a 0.050 2-tailed significance level, which was expected to be highly correlated with the primary endpoint, Nankivell creatinine clearance. Based on the actual number of enrolled and completed patients and the observed variability, there was approximately $80 \%$ power to detect this difference. Continuous data were expressed as means \pm SD and categorical data were expressed as numbers and percentages. The primary analysis was a $t$ test examining the difference in means between the 2 treatment groups. Fisher exact test was used for comparison of adverse events and other categorical variables, and one-way analysis of variance was used for continuous variables. Graft loss and patient death were analyzed using the Kaplan-Meier method for estimation of time to events. The log-rank test was used to assess statistical differences in the time-to-event analysis between groups. The distribution of histologic grade of first ARs in the 2 treatment groups was compared using a generalized Cochran-Mantel-Haenszel row mean score test. Differences in sirolimus and tacrolimus trough levels were analyzed via $t$ tests at each time point.

\section{RESULTS}

\section{Demographics and Baseline Characteristics}

Of the 128 enrolled patients, 63 were randomly assigned to rTAC and 65 were randomly assigned to sTAC. Patient demographics were similar between the two groups (Table 2). Recipients ranged in age from 18 to 78 years, $64.8 \%$ were male, and all were white. Overall, the most common primary etiologies for end-stage renal disease were glomerulonephritis $(32.8 \%)$ and polycystic kidney disease (13.3\%; Table 2). The mean number of human leukocyte antigen (HLA) mismatches and percentage of panelreactive antibodies were similar between groups.

\section{Immunosuppressive Drug Dosage and Trough Levels}

Mean sirolimus and tacrolimus whole blood trough levels and doses are depicted in Figs 1A and 1B. For both groups, sirolimus levels were maintained within the targeted range

\section{Table 2. Baseline Patient Characteristics ${ }^{a}$}

\begin{tabular}{lcc}
\hline \multicolumn{1}{c}{ Characteristic } & rTAC $(n=63)$ & sTAC $(n=65)$ \\
\hline Male, $n(\%)$ & $45(71.4)$ & $38(58.5)$ \\
White (\%) & 100 & 100 \\
Mean age $(\mathrm{y} \pm \mathrm{SD})$ & $47.9 \pm 13.3$ & $44.6 \pm 14.8$ \\
Deceased donor source, $n(\%)$ & $62(98.4)^{\mathrm{b}}$ & $65(100.0)$ \\
Primary transplant, $n(\%)$ & $54(85.7)$ & $60(92.3)$ \\
Mean ischemia time (h $\pm \mathrm{SD})$ & $17.6 \pm 6.6$ & $17.9 \pm 6.9$ \\
Mean HLA mismatches $\pm \mathrm{SD}$ & $2.9 \pm 1.3$ & $2.9 \pm 1.4$ \\
Mean panel reactive antibody (\% $\pm \mathrm{SD})$ & $2.5 \pm 7.3$ & $3.2 \pm 11.7$ \\
Delayed graft function, $n(\%)$ & $19(30.2)$ & $20(30.8)$ \\
Cause of end-stage renal disease, $n(\%)$ & & \\
Glomerulonephritis & $17(27.0)$ & $25(38.5)$ \\
Diabetes mellitus & $5(7.9)$ & $8(12.3)$ \\
Polycystic kidney disease & $11(17.5)$ & $6(9.2)$ \\
Hypertension & $3(4.8)$ & $5(7.7)$ \\
Interstitial disease & $5(7.9)$ & $4(6.2)$ \\
Other/unknown & $22(34.9)$ & $17(26.2)$ \\
\hline
\end{tabular}

rTAC, reduced-dose tacrolimus; sTAC, standard-dose tacrolimus; SD; standard deviation; HLA; human leukocyte antigen.

${ }^{a}$ No statistically significant differences between groups.

${ }^{\mathrm{b}}$ Donor source not recorded in 1 patient. 


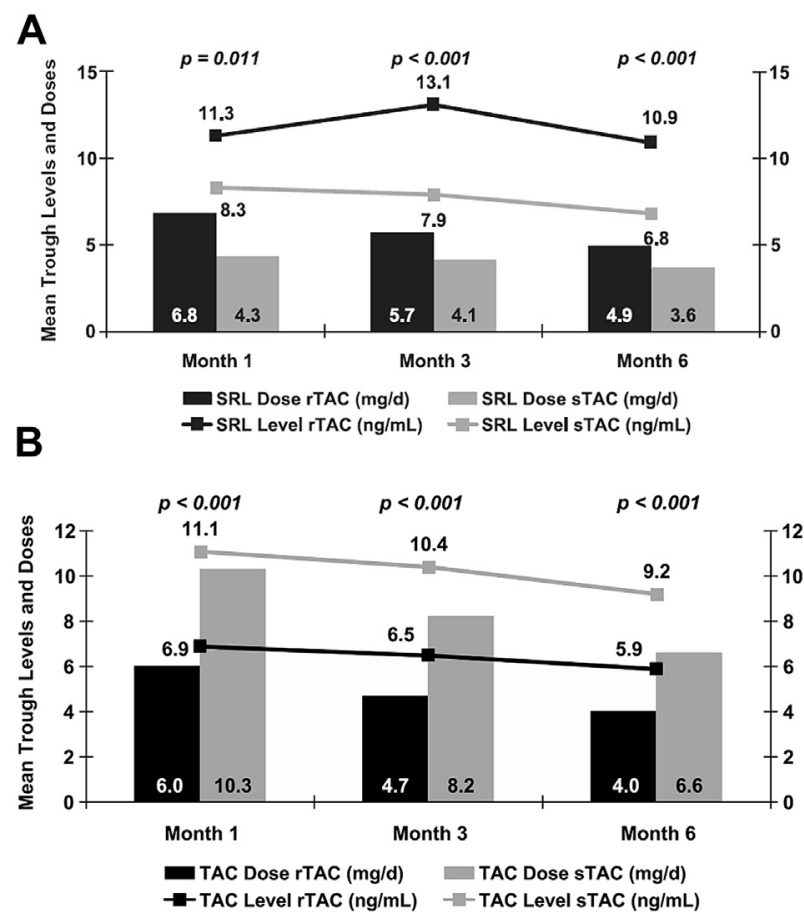

Fig 1. (A) Mean $S R L$, trough and dose. (B) Mean TAC trough and dose. $P$ value for comparison between rTAC versus STAC trough levels. SRL, sirolimus; TAC, tacrolimus; rTAC, reduceddose TAC plus SRL; sTAC, standard-dose TAC plus SRL.

(Table 1) at all time points, suggesting good protocol adherence. As expected, sirolimus levels were significantly higher $(P<.05)$ in the rTAC group than in the sTAC group at all time points. Mean tacrolimus levels for the rTAC group were maintained within the targeted range except at weeks 1 and 3, when they exceeded target levels by $0.2 \mathrm{ng} / \mathrm{mL}$ $(7.2 \pm 3.7 \mathrm{ng} / \mathrm{mL}$, target 3 to $7 \mathrm{ng} / \mathrm{mL})$ at week 1 , and by $0.1 \mathrm{ng} / \mathrm{mL}(7.1 \pm 3.7 \mathrm{ng} / \mathrm{mL}$, target 3 to $7 \mathrm{ng} / \mathrm{mL})$ at week 3 . Mean tacrolimus levels in the sTAC group were maintained within the targeted range throughout the study. As expected, the rTAC group exhibited significantly lower tacrolimus blood levels at all time points compared with the sTAC group $(P<.05)$.

Mean corticosteroid dose administration was consistent with the standard protocol taper regimen. Minor variations were observed across centers.

\section{Primary Endpoint}

In those patients remaining on therapy, mean creatinine clearance was significantly improved in the rTAC group by 3 months, and the differences between groups remained statistically significant for the duration of the study (Fig 2). By the primary endpoint of 6 months, rTAC patients exhibited significantly higher mean calculated creatinine clearance $(63.8 \pm 17.3 \mathrm{~mL} / \mathrm{min})$ than did sTAC patients $(52.7 \pm 18.9 \mathrm{~mL} / \mathrm{min}, P=.005)$.

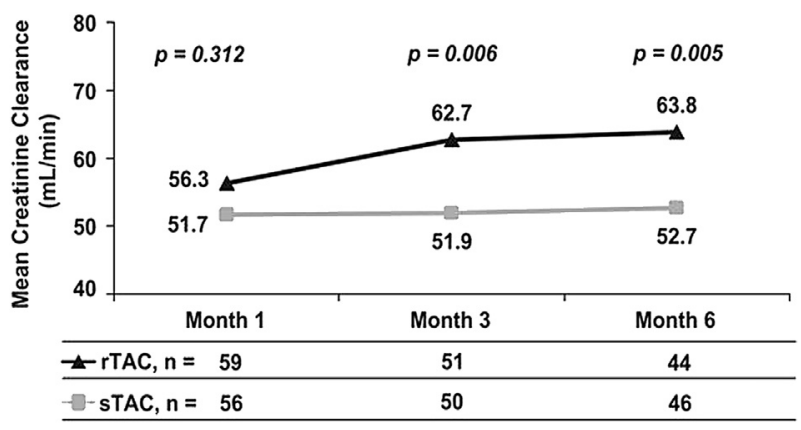

Fig 2. Mean calculated creatinine clearance. rTAC, reduceddose tacrolimus plus sirolimus; sTAC, standard-dose tacrolimus plus sirolimus.

\section{Serum Creatinine}

In patients remaining on therapy at 6 months, a trend toward lower mean serum creatinine in the rTAC group $(136.3 \pm 45.3 \mu \mathrm{mol} / \mathrm{L})$ compared with the sTAC group $(153.0 \pm 47.3 \mu \mathrm{mol} / \mathrm{L}, P=.085)$ was observed, although the difference did not reach statistical significance (Fig 3).

\section{Acute Rejection}

The overall incidence of AR was $17.5 \%(n=11)$ in the rTAC group and $7.7 \%(n=5)$ in the sTAC group $(P=.095)$. No statistically significant difference occurred between groups in the time to first BCAR. All rejections were mild to moderate in severity and responded to corticosteroid therapy. Of the 11 BCARs in the rTAC group, 4 were grade IA, 5 were grade IIA, and 2 were grade IIB. Among patients in the sTAC group with BCARs $(n=5)$, 4 were grade IA and 1 was grade IIB. Among patients experiencing BCARs, no difference was noted in donor source or in the presence or grade of chronic or sclerosing nephropathy between groups. Antibody therapy was used to treat rejection in 7 patients in the sTAC group and in 5 patients in the rTAC group. Additionally, 2 patients in the sTAC group and 2 patients in the rTAC group were treated for episodes considered rejections either without biopsy or graded as borderline by the investigator.

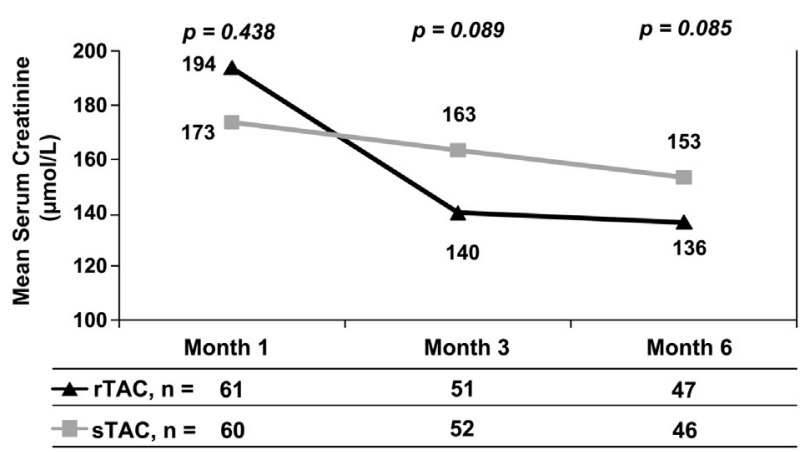

Fig 3. Mean serum creatinine. rTAC, reduced-dose tacrolimus plus sirolimus; STAC, standard-dose tacrolimus plus sirolimus. 
During the early study enrollment period, 6 BCARs occurred in the 19 patients randomly assigned to rTAC $(31.6 \%)$ and 0 BCARs occurred in the 17 patients enrolled in the sTAC group $(P=.023)$. All rejections occurred within the first 30 days after transplantation. Analysis revealed that subtherapeutic sirolimus and tacrolimus concentrations in the early post-transplant period may have contributed to the development of these rejections. A subsequent protocol amendment mandated an increase in sirolimus and tacrolimus dosages to ensure that targeted immunosuppressant levels were readily achieved early after transplantation. Target sirolimus and tacrolimus trough concentrations were not changed. After the amendment, the incidence of AR was similar between groups: rTAC, $11.4 \%(n=5 / 44)$; sTAC, $10.4 \%(n=5 / 48, P=.1)$.

\section{Patient and Graft Survival}

No significant differences in patient survival were observed between the rTAC and sTAC groups at 6 months posttransplantation $(95.2 \%$ vs $96.9 \%$, respectively; $P=.623)$. The causes of patient death were pulmonary hemorrhage, hemolytic uremic syndrome, and retroperitoneal hematoma in the rTAC group, and sepsis and cardiac arrest in the sTAC group.

Graft survival at 6 months post-transplantation was excellent in both the rTAC and sTAC groups $(93.7 \%$ vs $98.5 \%$, respectively; $P=.160$ ). Three patients in the rTAC group with BCARs subsequently lost their grafts. Of 5 patients in the sTAC group with BCARs, 4 maintained their grafts and 1 had no data available.

\section{Adverse Events and Discontinuations}

No significant differences were observed between treatment groups in the incidence of common adverse events, including hypertension, new-onset diabetes mellitus, or dyslipidemia (Table 3). Similarly, no statistically significant differences in infection rates occurred between groups (Table 4).

Overall, lipid parameters, including serum cholesterol and triglycerides, were similar between treatment groups throughout the study, except at screening, when serum cholesterol values differed between the sTAC and rTAC groups (5.4 vs $4.9 \mathrm{mmol} / \mathrm{L}$, respectively, $P=.046)$. The incidence of hypercholesteremia (13.8\% vs $12.7 \%$, respectively; $P=1.000)$ and hyperlipidemia $(23.1 \%$ vs $27.0 \%$, respectively; $P=.685$ ) were not significantly different between groups. The use of lipid-lowering therapy was similar between the sTAC (13.8\%) and rTAC (7.9\%) groups, as was the use of antihypertensives for blood pressure. Serum chemistries and hematologic parameters, including white blood cell and platelet counts, hemoglobin, and hematocrit, were similar between groups except for the period within 36 hours of hospital discharge, in which both white blood cell $\left(7.2\right.$ vs $\left.6.3 \times 10^{9} / \mathrm{L} ; P=.026\right)$ and platelet counts $\left(261.6\right.$ vs $\left.217.4 \times 10^{9} / \mathrm{L} ; P=.011\right)$ differed significantly between the sTAC and rTAC groups,
Table 3. Incidence of Selected Treatment-Emergent Adverse Events

\begin{tabular}{lcr}
\hline \multicolumn{1}{c}{ Adverse event, $n$ (\%) } & rTAC $(n=63)$ & sTAC $(n=65)$ \\
\hline Anemia & $18(28.6)$ & $17(26.2)$ \\
Hyperlipidemia & $17(27.0)$ & $15(23.1)$ \\
Hypercholesterolemia & $8(12.7)$ & $9(13.8)$ \\
Peripheral edema & $10(15.9)$ & $11(16.9)$ \\
Leukopenia & $10(15.9)$ & $9(13.8)$ \\
Thrombocytopenia & $9(14.3)$ & $7(10.8)$ \\
Arthralgia & $9(14.3)$ & $4(6.2)$ \\
Diarrhea & $8(12.7)$ & $17(26.2)$ \\
Vomiting & $8(12.7)$ & $10(15.4)$ \\
Nausea & $5(7.9)$ & $9(13.8)$ \\
ALT (SGPT) increased & $1(1.6)$ & $7(10.8)$ \\
AST (SGOT) increased & $0(0.0)$ & $6(9.2)^{\mathrm{a}}$ \\
Hyperglycemia & $3(4.8)$ & $8(12.3)$ \\
New-onset diabetes mellitus & $9(14.3)$ & $8(12.3)$ \\
Hypertension & $2(3.2)$ & $7(10.8)$ \\
\hline
\end{tabular}

ALT, alanine aminotransaminase; AST aspartate aminotransferase; rTAC, reduced-dose tacrolimus; STAC, standard-dose tacrolimus; SGPT, serum glutamic-pyruvic transaminase; SGOT, serum glutamic oxaloacetic transaminase. ${ }^{a} P<.05 ; P=$ not significant, all other comparisons.

respectively. No patients in the rTAC group experienced moderate $(>150 \mathrm{U} / \mathrm{L})$ or severe $(>500 \mathrm{U} / \mathrm{L})$ elevations in aspartate aminotransferase (AST). In the sTAC group, 1 patient (1.6\%, hepatitis B seropositive) had a moderate $(>150 \mathrm{U} / \mathrm{L})$ elevation in AST; no severe elevations in AST were observed. Moderate elevations $(>150 \mathrm{U} / \mathrm{L})$ in alanine transaminase (ALT) were observed in 3 patients $(4.8 \%$, 1 hepatitis B seropositive) in the rTAC group and in 5 patients $(7.9 \%)$ in the sTAC group. No elevations in AST or ALT ( $>150 \mathrm{U} / \mathrm{L})$ were noted among hepatitis C-seropositive patients. Two malignancies were reported: 1 patient in the rTAC group had a basal-cell carcinoma of the lip, and 1 patient in the sTAC group experienced posttransplant lymphoma.

Thirty-three (25.8\%) patients withdrew from the study: 15 $(23.8 \%)$ in the rTAC group and $18(27.7 \%)$ in the sTAC group $(P=.688)$. The primary reason for discontinuation was an adverse event: $11(17.5 \%)$ in the rTAC group and 14 $(21.5 \%)$ in the sTAC group. Four patients withdrew from the study because of lack of efficacy: 2 in the rTAC group

Table 4. Incidence of Treatment-Emergent Infections

\begin{tabular}{lcc}
\hline \multicolumn{1}{c}{ Infection, $n(\%)$} & rTAC $(n=63)$ & sTAC $(n=65)$ \\
\hline Candida & $2(3.2)$ & $4(6.2)$ \\
Sepsis & $1(1.6)$ & $3(4.6)$ \\
Cytomegalovirus & $3(4.8)$ & $5(7.7)$ \\
Pneumonia & $2(3.2)$ & $6(9.2)$ \\
Herpes simplex & $1(1.6)$ & $0(0.0)$ \\
Herpes zoster & $0(0.0)$ & $1(1.5)$ \\
Urinary tract infection/pyelonephritis & $8(12.7)$ & $3(4.6)$ \\
Lymphocele & $6(9.5)$ & $7(10.8)$ \\
Dehiscence & $3(4.8)$ & $1(1.5)$ \\
Wound infection & $1(1.6)$ & $1(1.5)$ \\
\hline
\end{tabular}

$P=$ not significant, all comparisons. rTAC, reduced-dose tacrolimus; STAC, standard-dose tacrolimus. 
and 2 in the sTAC group. In the rTAC group, both cases resulted in AR and graft loss, 1 at day 9 and 1 at day 34. In the sTAC group, both cases resulted in AR and graft loss, 1 at day 3 and 1 at day 71. Specific reasons for withdrawal in the sTAC group included infection $(n=3$, including 2 infectious pneumonias), tacrolimus nephrotoxicity $(n=2)$, gastrointestinal complaints $(n=2)$, withdrawal of consent $(n=2)$, graft loss due to thrombosis $(n=1)$, hypersensitivity to tacrolimus $(n=1)$, tremor $(n=1)$, polyneuropathy $(n=1)$, bilateral pain in heels $(n=1)$, pneumopathy $(n=1)$, and post-transplant lymphoma $(n=1)$. Similarly, specific reasons for withdrawal in the rTAC group included infection $(n=2$, including 1 pseudomonas pneumonia and 1 endocarditis), wound complications ( $n=2$, including dehiscence and lymphocele developing an abscess), sirolimus intolerance $(n=2)$, arthralgia $(n=1)$, voluntary withdrawal $(n=1)$, hypertriglyceridemia $(n=1)$, tubulointerstitial nephritis $(n=1)$, pneumonitis $(n=1)$, synovitis $(n=1)$, and death $(n=1)$. Of those who withdrew early, sTAC patients exhibited significantly higher baseline total cholesterol values compared with rTAC patients $(5.5 \pm$ 1.23 vs $4.3 \pm 0.97 \mathrm{mmol} / \mathrm{L}$, respectively; $P=.024)$ and had significantly more HLA mismatches $(3.2 \pm 0.99$ vs $2.3 \pm$ 1.05, respectively; $P=.025)$.

\section{DISCUSSION}

The results from this prospective, randomized trial demonstrate that, in the absence of antibody induction, a regimen of rTAC and corticosteroids in low- to moderaterisk renal allograft recipients provides adequate prophylaxis against $\mathrm{AR}$ and is associated with significantly improved renal allograft function, based on the primary endpoint of this study, creatinine clearance ${ }^{27}$ at 6 months. The improvement in renal function observed in the rTAC cohort was not unexpected. The use of CNIs at therapeutic doses sufficient to prevent allograft rejection is known to reduce glomerular filtration rates by approximately $15 \%$ to $25 \%$ and may lead to tubulointerstitial fibrosis. ${ }^{28-30}$ The improvement in glomerular filtration rate among rTAC patients was observed despite a trend toward a higher incidence of BCARs in this group and a trend toward lower mean serum creatinine in the rTAC group compared with the sTAC group, although the difference was not significant.

Renal function within the first year after transplantation may be an important factor influencing graft survival. ${ }^{31-34}$ From the analysis of Hariharan et al, renal allograft recipients with a serum creatinine level of $1.5 \mathrm{mg} / \mathrm{dL}$ at 1 year and/or a change in creatinine $\geq 0.3 \mathrm{mg} / \mathrm{dL}$ between months 6 and 12 after transplantation have a substantially lower projected graft half-life than all other groups, regardless of prior AR. ${ }^{34}$ Although the duration of follow-up in our study was only 6 months, the rTAC group experienced a significant improvement in Nankivell creatinine clearance.

When an early increased incidence of AR was identified in the rTAC group, analysis revealed that this was likely due to underachieved target levels of sirolimus and tacrolimus in the early postoperative period, prompting a protocol amendment to increase the initial dosage of both sirolimus and tacrolimus to ensure rapid achievement of targeted concentrations of both agents. Of note, the protocolmandated target concentrations for sirolimus and tacrolimus were not changed; additionally, it should be noted that the amendment change did not result in significantly higher immunosuppressant trough levels, as overall mean target levels essentially remained within range throughout the duration of the study. Following the protocol amendment, the adjustment of the initial dosages of sirolimus and tacrolimus proved effective in AR prophylaxis, as indicated by a similar incidence of BCARs between treatment groups. These results emphasize the importance of optimal sirolimus and tacrolimus exposure to achieve adequate trough levels of both drugs in the early post-transplant period.

A fundamental objective of combining the two immunosuppressants was to reduce dosages of both drugs with the goal of improving patient compliance and decreasing adverse events while maintaining effective prophylaxis against AR. The combination of sirolimus and tacrolimus is known to exhibit immunosuppressive synergy. ${ }^{15}$ Numerous single-center reports describe sirolimus/tacrolimus-based immunosuppression in organ transplant recipients. ${ }^{17-25}$ Consistent with the findings of our study, a retrospective analysis by El-Sabrout et $\mathrm{al}^{19}$ emphasized the use of sirolimus loading doses to increase rejection-free survival. In a pilot study of early tacrolimus withdrawal, Grinyo et al reported improved renal function and blood pressure in patients randomly assigned to a regimen of tacrolimus withdrawal versus those who remained on standard-dose tacrolimus and sirolimus. ${ }^{24}$ These investigators also highlighted the importance of achieving target sirolimus and tacrolimus levels in the early period after transplantation. These findings of CNI withdrawal are further supported by the results of the Rapamune Maintenance Regimen trial that demonstrated improved renal allograft survival, renal function, and blood pressure after early cyclosporine withdrawal and sirolimus maintenance therapy. ${ }^{35}$ An alternative strategy to limit AR may include the use of antibody induction. Early experience using antibody induction followed by reduced tacrolimus and sirolimus dosages has demonstrated success in minimizing the risk of rejection, ${ }^{36,37}$ but these benefits must be weighed against the potential for increased risk of infection and/or posttransplant lymphoproliferative disease.

Contrary to the results of this study, an analysis by MeierKriesche et al of 44,915 adult renal transplant recipients from the Scientific Renal Transplant Registry concluded that the combination of sirolimus and tacrolimus is associated with significantly worse graft survival compared with tacrolimus plus mycophenolate mofetil (MMF). It is important to note that this analysis was conducted using a retrospective database. ${ }^{38}$ The results from the present study indicate that the combination of tacrolimus and sirolimus was generally well tolerated. No unexpected or unusually pronounced adverse events were identified, and 
concomitant use of rTAC and sirolimus did not appear to place patients at increased risk for specific adverse events.

Importantly, the use of sirolimus to minimize chronic tacrolimus exposure may have the potential to reduce the incidence and severity of tacrolimus-associated adverse events, such as nephrotoxicity and hypertension. No statistically significant differences were observed between groups in the incidence, type, or severity of adverse events, including hematologic toxicity (leukopenia, thrombocytopenia, anemia), hypertension, dyslipidemia, infection (including pneumonia), wound complications (including lymphocele and dehiscence), and malignancy. While not statistically significant, there were increased incidences of arthralgia and urinary infection in the rTAC groups and diarrhea, nausea, hyperglycemia, hypertension, and increases in AST/ALT in the STAC groups; from a clinical perspective, these warrant consideration, as do any known adverse effects with sirolimus and tacrolimus. Gonwa et al reported that the combination of sirolimus and standarddose tacrolimus may exacerbate nephrotoxicity and result in inferior renal function and higher blood pressure compared with MMF and standard-dose tacrolimus. ${ }^{22}$ Lo et al also reported a high incidence of biopsy-proven tacrolimus nephrotoxicity with the combined use of full doses of tacrolimus and sirolimus. ${ }^{17}$ Furthermore, tacrolimus generally has a less significant impact on lipids, a known adverse effect of sirolimus compared with cyclosporine. ${ }^{39,40}$

A limitation of this study was that it was not adequately powered to accurately assess the risk of AR. The study population was generally considered to be of low immunologic risk, predominantly consisting of nonsensitized white, European recipients of a primary allograft. Prospective protocol biopsies were not performed and, therefore, we cannot draw any conclusions about the incidence or influence of subclinical rejection or if observed improvements in renal function correlated with improved histology. The potential benefits of improved renal allograft function as observed in the rTAC group must be weighed against the potential risk for AR.

In conclusion, the results of this prospective randomized trial demonstrate that, in a population of low- to moderaterisk renal allograft recipients, a regimen of sirolimus, rTAC, and corticosteroids achieves superior renal function while maintaining an acceptable incidence of ARs in the first 6 months after transplantation compared with a regimen of sirolimus, sTAC, and corticosteroids. When using a regimen of rTAC plus sirolimus and corticosteroids in the absence of antibody induction, it is critical to ensure that therapeutic levels of sirolimus and tacrolimus are achieved early in the postoperative period to minimize the risk of early AR. The observations from our study suggest that the balance between efficacy and toxicity obtained with this combination may be more favorable if tacrolimus levels are further lowered or if tacrolimus is eliminated altogether. Further study with longer follow-up will be necessary to fully characterize the safety of this immunosuppressive combination and to determine if the benefit of improved renal function observed in this study correlates with improved long-term graft survival.

\section{ACKNOWLEDGMENTS}

This study was sponsored by Wyeth Pharmaceuticals, which was acquired by Pfizer Inc in October 2009. No author received an honorarium or other form of financial support related to the development of this manuscript. Medical writing support was provided by Susan A. Nastasee and Sara Parambil, PharmD, of Wyeth. Additional editorial support was provided by Bina J. Patel, PharmD, of Peloton Advantage and was funded by Pfizer Inc.

The following investigators also participated in this trial as part of the European Rapamune Tacrolimus Study Group: John Boletis (Nephrology Clinic, Laikon General Hospital, Athens, Greece); Marco Castagneto (Università Cattolica, Rome, Italy); Johann Hauss (Universitat Leipzig, Leipzig, Germany); Ulrich Kunzendorf (Universität Erlangen/Nürnberg, Erlangen/Nürnberg, Germany); Peter Neuhaus (Universitätsklinikum Charité Campus Virchow, Berlin, Germany); Rainer Oberbauer (Medical University of Vienna, Vienna, Austria); Giuseppe Segoloni (Azienda Ospedaliera S. Giovanni Battista Hospital, Turin, Italy); Hans-Krister Stummvoll ${ }^{\dagger}$ (deceased; Hospital Elisabethinen, Linz, Austria); and Yves Vanrenterghem (Universitair Ziekenhuis Gasthuisberg, Leuven, Belgium).

\section{REFERENCES}

1. Nankivell BJ, Borrows RJ, Fung CL, et al. The natural history of chronic allograft nephropathy. N Engl J Med. 2003;349:2326.

2. Bennett WM, DeMattos A, Meyer MM, et al. Chronic cyclosporine nephropathy: the Achilles' heel of immunosuppressive therapy. Kidney Int. 1996;50:1089.

3. Solez K, Vincenti F, Filo RS. Histopathologic findings from 2-year protocol biopsies from a U.S. multicenter kidney transplant trial comparing tacrolimus versus cyclosporine: a report of the FK506 Kidney Transplant Study Group. Transplantation. 1998;66: 1736.

4. Davies DR, Bittmann I, Pardo J. Histopathology of calcineurin inhibitor-induced nephrotoxicity. Transplantation. 2000;69(Suppl 12):SS11.

5. Andoh TF, Burdmann EA, Bennett WM. Nephrotoxicity of immunosuppressive drugs: experimental and clinical observations. Semin Nephrol. 1997;7:34.

6. Sehgal SN. Rapamune (Sirolimus, rapamycin): an overview and mechanism of action. Ther Drug Monit. 1995;17:660.

7. Terada N, Lucas JJ, Szepesi A, et al. Rapamycin blocks cell cycle progression of activated $\mathrm{T}$ cells prior to events characteristic of middle to late G1 phase of the cycle. J Cell Physiol. 1993;154:7.

8. Marx SO, Jayaraman T, Go LO, et al. Rapamycin-FKBP inhibits cell cycle regulators of proliferation in vascular smooth muscle cells. Circ Res. 1995;76:412.

9. Poon M, Marx SO, Gallo R, et al. Rapamycin inhibits vascular smooth muscle cell migration. J Clin Invest. 1996;98:2277.

10. Gregory CR, Huie P, Billingham ME, et al. Rapamycin inhibits arterial intimal thickening caused by both alloimmune and mechanical injury. Its effects on cellular, growth factor, and cytokine response in injured vessels. Transplantation. 1993;55:1409.

11. Gallo R, Padurean A, Jayaraman T, et al. Inhibition of intimal thickening after balloon angioplasty in porcine coronary arteries by targeting regulators of the cell cycle. Circulation. 1999;99:2164.

12. Sousa JE, Costa MA, Abizaid A, et al. Lack of neointimal proliferation after implantation of sirolimus-coated stents in human coronary arteries: a quantitative coronary angiography and threedimensional intravascular ultrasound study. Circulation. 2001;103:192. 
13. Dumont FJ, Melino MR, Staruch MJ, et al. The immunosuppressive macrolides FK-506 and rapamycin act as reciprocal antagonists in murine T cells. J Immunol. 1990;144:1418.

14. Bierer BE, Mattila PS, Standaert RF, et al. Two distinct signal transmission pathways in $\mathrm{T}$ lymphocytes are inhibited by complexes formed between an immunophilin and either FK506 or rapamycin. Proc Natl Acad Sci U S A. 1990;87:9231.

15. Vu MD, Qi S, Xu D, et al. Tacrolimus (FK-506) and sirolimus (rapamycin) in combination are not antagonistic but produce extended graft survival in cardiac transplantation in the rat. Transplantation. 1997;64:1853.

16. Sigal NH, Dumont FJ, Cyclosporin A. FK506, and rapamycin: pharmacologic probes of lymphocyte signal transduction. Annu Rev Immunol. 1992;10:519.

17. Lo A, Egidi MF, Gaber LW, et al. Observations regarding the use of sirolimus and tacrolimus in high-risk cadaveric renal transplantation. Clin Transplant. 2004;18:53.

18. Lo A, Egidi MF, Gaber LW, et al. Comparison of sirolimusbased calcineurin inhibitor-sparing and calcineurin inhibitor-free regimens in cadaveric renal transplantation. Transplantation. 2004; $77: 1228$

19. El-Sabrout R, Delaney V, Butt F, et al. Improved freedom from rejection after a loading dose of sirolimus. Transplantation. 2003;75:86.

20. Russ GR, Campbell S, Chadban S, et al. Reduced and standard target concentration tacrolimus with sirolimus in renal allograft recipients. Transplant Proc. 2003;35(suppl 3):115S.

21. Van Hooff JP, Squifflet JP, Wlodarczyk Z, et al. A prospective randomized multicenter study of tacrolimus in combination with sirolimus in renal-transplant recipients. Transplantation. 2003;75:1934.

22. Gonwa T, Mendez R, Yang HC, et al. Randomized trial of tacrolimus in combination with sirolimus or mycophenolate mofetil in kidney transplantation: results at 6 months. Transplantation. 2003;75:1213.

23. Ciancio G, Burke GW, Gaynor JJ, et al. A randomized longterm trial of tacrolimus/sirolimus versus tacrolimus/mycophenolate mofetil versus cyclosporine (Neoral)/sirolimus in renal transplantation. II. Survival, function, and protocol compliance at 1 year. Transplantation. 2004;77:252.

24. Grinyo JM, Campistol JM, Paul J, et al. Pilot randomized study of early tacrolimus withdrawal from a regimen with sirolimus plus tacrolimus in kidney transplantation. Am J Transplant. 2004;4:1308.

25. McAlister VC, Gao Z, Peltekian K, et al. Sirolimus-tacrolimus combination immunosuppression. Lancet. 2000;355:376.

26. Solez K, Axelsen RA, Benediktsson H, et al. International standardization of criteria for the histologic diagnosis of renal allograft rejection: the Banff working classification of kidney transplant pathology. Kidney Int. 1993;44:411.

27. Nankivell BJ, Gruenewald SM, Allen RD, et al. Predicting glomerular filtration rate after kidney transplantation. Transplantation. 1995;59:1683.

28. de Mattos AM, Olyaei AJ, Bennett WM. Nephrotoxicity of immunosuppressive drugs: long-term consequences and challenges for the future. Am J Kidney Dis. 2000;35:333.

29. Ong AC, Fine LG. Loss of glomerular function and tubulointerstitial fibrosis: cause or effect? Kidney Int. 1994;45:345.

30. Rowshani AT, Scholten EM, Bemelman F, et al. No difference in degree of interstitial Sirius red-stained area in serial biopsies from area under concentration-over-time curves-guided cyclosporine versus tacrolimus-treated renal transplant recipients at one year. J Am Soc Nephrol. 2006;17:305.

31. Cecka MJ. The UNOS Scientific Renal Transplant Registry. In: Cecka MJ, Terasaki PI, eds. Clinical Transplants 1998. Los Angeles, CA: UCLA Tissue Typing Laboratory; 1998:1-16.

32. Cosio FG, Pelletier RP, Falkenhain ME, et al. Impact of acute rejection and early allograft function on renal allograft survival. Transplantation. 1997;63:1611.

33. Nicol D, MacDonald AS, Belitsky P. Early prediction of renal allograft loss beyond one year. Transplant Int. 1993;6:153.

34. Hariharan S, McBride MA, Cherikh WS, et al. Post-transplant renal function in the first year predicts long-term kidney transplant survival. Kidney Int. 2002;62:311.

35. Oberbauer R, Segoloni G, Campistol JM, et al. Early cyclosporine withdrawal from a sirolimus-based regimen results in better renal allograft survival and renal function at 48 months after transplantation. Transplant Int. 2005;18:22.

36. Lawen J, Keough-Ryan T, Clase C, et al. Sirolimus and lowdose tacrolimus with antibody induction in kidney transplantation: preliminary results of a pilot study. Transplant Proc. 2001;33:3223.

37. Salazar A, McAlister VC, Kiberd BA, et al. Sirolimustacrolimus combination for combined kidney-pancreas transplantation: effect on renal function. Transplant Proc. 2001;33:1038.

38. Meier-Kriesche H, Schold JD, Srinivas TR, et al. Sirolimus in combination with tacrolimus is associated with worse renal allograft survival compared to myocophenolate mofetil combined with tacrolimus. Am J Transplant. 2005;5:2273-2280.

39. Pirsch JD, Miller J, Deier MH, et al. A comparison of tacrolimus (FK506) and cyclosporine for immunosuppression after cadaveric renal transplantation. Transplantation. 1997;63:977.

40. Jensik. SC and the FK Kidney Transplant Study Group. Tacrolimus (FK 506) in kidney transplantation: three-year survival results of the US multicenter, randomized, comparative trial. Transplant Proc. 1998;30:1216. 